\title{
PIAUILISMO: PROPAGADOR DO PATRIMÔNIO CULTURAL PIAUIENSE ATRAVÉS DA MEMÓRIA DIGITAL
}

\author{
Cristhianne Castro da Silva ${ }^{1}$
}

ORCID: 0000-0002-6396-3192

Artemísia lima Caldas ${ }^{2}$

ORCID: 0000-0002-9532-1784

Resumo: Piauilismo, desfile de moda dos formandos do Curso de Moda, Design e Estilismo da Universidade Federal do Piauí, tem como objetivos específicos elencar a importância do evento como propagador de cultura, demonstrar como as mídias sociais preservam viva a cultura propagada e comprovar que as memórias digitais favorecem a divulgação do mesmo como propagador do patrimônio cultural piauiense. Nisso, baseando-se em elementos da cultura local, valorizando o patrimônio cultural piauiense em todos os aspectos que a pesquisa possibilita na criação. Neste trabalho, relatamos sobre a produção do Piauilismo nos anos de 2013 a 2018, a partir de pesquisas com a combinação dos métodos de levantamento de dados, através de entrevistas e questionários com pessoas envolvidas no projeto. Ainda,

1 Pós graduanda em Moda, Cultura e Mercado pela Faculdade Adelmar Rosado - FAR, Bacharel em Design da Moda e Estilismo pela Universidade Federal do Piauí - UFPI. E-mail: (thianneartes@ gmail.com).

2 Doutora em Engenharia Têxtil (2017) Escola de Engenharia da Universidade do Minho (UMinho), Mestra em Avaliação de Políticas Públicas (2011) Universidade Federal do Ceará (UFC), Especialista em Design Têxtil em Moda (2006) Faculdade Católica do Ceará (FCC), Bacharela em Estilismo e Moda (2002) UFC e Bacharela (1991) em Administração de Empresas (1991) Universidade de Fortaleza (UNIFOR). Docente de 2002 a 2006 no Curso de Design de Moda-UFC e na FCC de 2005 a 2009. Desde de 2009 docente do curso de Moda, Design e Estilismo da Universidade Federal do Piauí (UFPI). Experiências em projetos sociais correlacionados com o design de moda, atuando nos seguintes temas: ergonomia do vestuário, desenvolvimento de produtos de moda inclusiva, artesanato e tecnologia do vestuário conexo a modelagem do vestuário. E-mail: (artecaldas2@gmail.com). 
apresentamos um projeto de extensão e as possibilidades de acesso dos materiais expostos através das redes sociais como forma de divulgar a produção acadêmica e a valorização da memória do patrimônio cultural.

Palavras-chave: Patrimônio cultural. Memória. Redes sociais. Produção de Moda.

\title{
PIAUILISMO: PROPAGATOR OF PIAUIENSE CULTURAL HERITAGE THROUGH DIGITAL MEMORY
}

\begin{abstract}
Piauilismo, fashion show of the graduates of the Fashion, Design and Styling Course of the Federal University of Piauí, has as specific objectives to list the importance of the event as a propagator of culture, demonstrate how the social media preserve alive the propagated culture and prove that the digital memories favor the dissemination of the same as propagator of the cultural heritage of Piaui. In this, based on elements of local culture, valuing the Piauiense cultural heritage in all aspects that research makes possible in creation. In this work, we report on the production of Piauilismo in the years 2013 to 2018, from surveys with the combination of data collection methods, through interviews and questionnaires with people involved in the project. Also, we present an extension project and the possibilities of access to the materials exposed through social networks as a way to disseminate academic production and the appreciation of the memory of cultural heritage.
\end{abstract}

Keywords: Cultural heritage. Memory. Social networks. Fashion Production.

\section{PIAUILISMO: PROPAGADOR DEL PATRIMONIO CULTURAL PIAUIENSE ATRAVÉS DE LA MEMORIA DIGITAL}

Resumen: Piauilismo, desfile de moda de los graduados del Curso de Moda, Diseño y Estilismo de la Universidad Federal de Piauí, tiene como objetivos específicos listar la importancia del evento como propagador de la cultura, demostrar cómo los medios sociales preservan viva la cultura propagada y demostrar que las memorias digitales favorecen la difusión de la misma como propagador del patrimonio cultural de Piauí. En esto, a partir de elementos de la cultura local, valorando el patrimonio cultural Piauiense en todos los aspectos que la investigación hace posible en la creación. En este trabajo, informamos sobre la producción de Piauilismo en los años 2013 a 2018, desde encuestas con la combinación de métodos de recolección de datos, pasando por entrevistas y cuestionarios con personas involucradas en el proyecto. Asimismo, se presenta un proyecto de ampliación y las posibilidades de acceso a los materiales expuestos a través de las redes sociales como forma de difundir la producción académica y la valorización de la memoria del patrimonio cultural.

Palabras claves: Patrimonio cultural. Memoria. Redes sociales. Producción de Moda. 


\section{INTRODUÇÃO}

O curso de Bacharelado em Moda, Design e Estilismo - CMDE, da Universidade Federal do Piauí - UFPI, desde o ano de 2013 tem como proposta de conclusão do curso, a produção de um desfile de moda e curadoria que vem sendo desenvolvida pelos discentes que estão cursando o nono período, sendo que este evento busca enfatizar a pesquisa sobre o patrimônio cultural piauiense, que é mostrado para a comunidade no ato criativo de jovens talentos no mercado de moda de Teresina e sua divulgação através das mídias sociais, tais como o Facebook, Instagram, Whats App e Youtube. Além disso, o evento é realizado em locais públicos e de fácil acesso para que os admiradores, publicitários e empresários para que possam vislumbrar o resultado do trabalho que carrega a identidade do povo, através da indumentária e a produção de moda.

O presente artigo busca mostrar a importância das mídias sociais para a divulgação do patrimônio cultural piauiense apresentado no Piauilismo, que a reprodução iconográfica está adequada a natureza conceitual do conhecimento histórico, através dos recursos aliados ao conjunto das técnicas relacionadas à fotografia (CHOAY, 2001, p. 233), maneira pela qual os recursos que comprovam a importância do evento de moda como propagador de cultura através das fontes imagéticas, sejam as fotos ou vídeos.

Nesse sentido, questiona-se sobre qual a importância das memórias digitais divulgadas pelas mídias sociais ao relatarem os elementos culturais piauiense através dos registros associados ao Piauilismo. Para tanto, buscou-se referências em Matharu (2011) que defende alguns aspectos acerca das produções realizadas dentro de um curso de design de moda e sobre a produção de moda, o qual aponta que "[...]. A roupa também pode associar quem a veste a um grupo específico com ideias, gostos, origens, culturas e religiões semelhantes. (MATHARU, 2011, p.8).

Esta pesquisa vem a apresentar o processo de construção da imagem do Piauilismo, evento de moda dos formandos do CMDE, da UFPI como um propagador do patrimônio cultural através da produção da indumentária e elementos compositivos dos espaços utilizados para a realização de desfiles de moda. A ideia principal deste projeto de extensão acadêmica "é enaltecer os aspectos culturais, regionais e local evidenciando o estado do Piauí e suas riquezas como fonte de inspiração para coleções de moda" (CARVALHO e SOUSA, 2019, p.9). 
Desta forma, esse artigo mostra como o projeto de extensão ligado ao processo produtivo do Piauilismo move toda a comunidade acadêmica do CMDE, no intuito de possibilitar os discentes que estão no processo produtivo das peças comerciais e autorais, a observarem o que há de valor desde a produção dos materiais artesanais, o modo de fazer, as características que cada matéria-prima utilizada na confecção de peças autorais que possuem valores intrínsecos que caracterizam a cultura piauiense, que tange nos aspectos da imaterialidade, pelo ato do fazer, e a abordagem da materialidade com o produto confeccionado e mostrado para a sociedade que anseia por conhecimento ou mesmo a curiosidade.

Nesse sentido este artigo busca inserir informações pertinentes a pesquisa associada as mídias sociais que se tornaram importantes veículos de divulgação e ao mesmo tempo um elemento extremamente importante para o registro gerado pelas memórias digitais, pois ao colocarmos em sites de busca ou até no site da instituição que surgiu o Piauilismo, pode-se acessar informações através de textos, imagens e vídeos que mostram os detalhes de cada uma das edições já produzidas pelos discentes do curso de Moda, as quais acontecem na cidade de Teresina em pontos estratégicos valorizando a história da cidade.

No entanto, a produção deste material de apoio deve-se as pesquisas, como abordado anteriormente, através do site da UFPI, Facebook, Instagram e no Youtube através do canal UFPI TV e WhatsApp, que por meio dessas mídias sociais e digitais há registros desde aos envolvidos no processo de construção da imagem da edição do Piauilismo, em que foi possível registrar as dez primeiras edições do evento, e materiais de apoio como livros e artigos associados a moda, ao patrimônio e o resgate da memória através das mídias digitais que colaboram para o registro dos eventos promovidos pelo projeto de extensão em questão.

Tendo-se neste artigo como objetivo principal o de relatar como o Piauilismo divulga o patrimônio cultural piauiense através das memórias digitais, buscando-se neste sentido elencar a importância do evento como divulgador de cultura, demonstrar como as mídias sociais preservam viva a cultura propagada por ele, e, por último comprovar que as memórias digitais favorecem a divulgação do Piauilismo por se tratar dos aspectos intrínsecos ao patrimônio cultural piauiense.

Para o desenvolvimento da pesquisa foi aplicada a combinação dos métodos, levantamento bibliográfico e pesquisas de campo com coleta de dados, através de entrevistas com pessoas envolvidas na produção do evento Piauilismo. Esse levantamento foi necessário para entender como é trabalhado a 
inter-relação entre o patrimônio histórico-artístico-cultural e a moda desenvolvida pelos discentes da UFPI, entre os anos de 2013 a 2018.

Para o levantamento bibliográfico fez-se necessário a utilização de livros, artigos, e-books e sites, além das mídias sociais ou fontes digitais. O referencial teórico consiste na apresentação de como a moda relaciona-se com a cultura e o patrimônio cultural, em conjunto com os desdobramentos adquiridos por pesquisas realizadas anteriormente, tendo como base os relatos das experiências dos envolvidos no processo de pesquisa de criação e de desenvolvimento de produtos para o Piauilismo. Foi evidenciado o levantamento histórico do desenvolvimento das dez edições do evento.

Com relação as fontes digitais pesquisadas, foi possível extrair imagens, dando a possibilidade de se vislumbrar a apresentação visual do evento, como a forma da passarela, os tipos de iluminação, as roupas desenvolvidas, a ornamentação do ambiente, entre outros detalhes. Neste sentido, foi feita uma análise da comunicação visual estabelecida pelos desfiles de moda promovidos pelo CMDE, o qual é responsável por dar notoriedade aos futuros designers de moda do Piauí, que em sua essência, buscam sempre evidenciar os aspectos intrínsecos as riquezas do patrimônio cultural existente no estado.

As pesquisas anteriormente realizadas para coleta de todos os dados, iniciou-se com a organização de diversos trabalhos realizados dentro do projeto de extensão do Piauilismo, em que equipes de alunos participantes das edições supracitadas, depois organizadas em partes, para melhor compreensão, e focou-se nos aspectos históricos e sociológicos sobre como são importantes as relações entre os desfiles de moda e a sociedade, os quais as mídias sociais digitais preservam viva a cultura piauiense.

\section{PIAUILISMO E A PRESERVAÇÃO DA MEMÓRIA}

No ano de 2013, a primeira turma do Curso de Moda, Design e Estilismo CMDE da Universidade Federal do Piauí - UFPI estava em fase de conclusão, assim como em diversos cursos da área de moda no último ano, os projetos são desenvolvidos de forma intensiva, como uma maneira de explorar as ideias, possibilitar aos discentes a interação com os materiais e aperfeiçoar técnicas aprendidas ao longo do curso, sendo assim "a coleção final de curso deve demonstrar que o aluno pode manter um envolvimento sólido com a condução e a resolução de seus trabalhos e que é capaz de criar com autonomia e de acordo com padrões profissionais". (JONES, 2011, p.15) 
Professor Ascânio Wanderley, responsável pelas disciplinas de projeto de coleção I e II, coordena o processo de construção da imagem do Piauilismo, fazendo com que o estado do Piauí seja visto numa outra perspectiva e que possa ser valorizada a cultura local, dessa forma cada semestre de turma de formandos ficam responsáveis em produzirem um briefing dentro da temática escolhida pelo coordenador do projeto, assim é feito o acompanhamento do processo criativo para o desenvolvimento das coleções comerciais e autorais, sendo possível perceber que:

\begin{abstract}
A importância destas coleções, com a apresentação de várias temáticas piauienses, é de estimular as percepções de desenvolvimento de produto de vestuário evidenciando a cultura local em busca de uma identidade de moda com foco na produção intelectual, artesanal, cultural, social e econômica do estado do Piauí. Com este propósito, buscam-se parcerias para desenvolver cada vez mais este projeto para que se divulgue um Piauí de excelência. (CARVALHO e SOUSA, 2019, p.11)
\end{abstract}

E dentro das parcerias que divulgam o Piauilismo, pode-se destacar os meios de comunicação, tais como: programas televisivos locais, as emissoras de rádio, panfletos de divulgação nos espaços da Universidade, as mídias sociais digitais (Facebook, Instagram, Youtube e WhatsApp) estimulando um grande número de pessoas a terem acesso à informação do que é o evento, onde e quando será realizado, além da temática que será destacada no aspecto regionalista piauiense.

A importância dessas divulgações para o Piauilismo dar-se-á pelos desdobramentos de interlocuções, ao tratarmos a questão da identidade do evento acadêmico, que através da internet tem ganhado grandes proporções no mercado local, pois a internet e os demais meios de comunicação percebem a importância e o alcance da divulgação do mesmo. Observa-se também que histórias são contadas a partir do momento que o público interage com as publicações disponibilizadas no meio digital. Sendo importante destacar que

A internet é um espaço de narrativas: histórias do cotidiano e da memória do presente.

É um lugar de histórias, ideias e opiniões. E, as redes sociais são a face mais visível desse fenômeno porque além de sua configuração original como espaços de interação social, de troca de afetos e de comunicação, elas passaram a ocupar uma espécie de um lugar digital de memória. (HENRIQUES, 2017, p.125).

Ao fazer um apanhado das dez primeiras edições, que compreende os anos de 2013 a 2018 foi possível resgatar informações pertinentes sobre as temáticas trabalhadas em cada uma das coleções apresentadas pelos formandos, reafirmando assim que o espaço digital é extremamente importante para o resgate da memória 
de um evento que culmina no fechamento de um ciclo de aprendizagem e de valorização cultural, além de divulgar os novos designers do mercado local.

Destacando a memória no contexto histórico do Piauilismo foi possível perceber que os participantes que foram entrevistados para a coleta de dados sobre as temáticas e como se deu o processo de produção da indumentária, cenografia e a abordagem do tema proposto por Ascânio, nota-se que muitos dos ex- acadêmicos do curso, ao responderem o questionário tiveram dificuldades de responder alguns itens, assim confirmamos que nossa memória é seletiva e que selecionamos aquilo que consideramos ou não importante ao longo de nossas vidas.

"As memórias registradas em livros, ou mesmo através da internet, não podem ser dissociadas de um processo friccional, pois ninguém recorda e registra exatamente como aconteceu, mas o que restou da lembrança e esquecimento do que se passou". (HENRIQUES, 2017, p. 128). A internet, através dos registros das mídias digitais está cheia de memórias preservadas e que ao abrirmos um site de busca com o nome "Piauilismo", vem um apanhado de fotografias, relatos jornalísticos, vídeos e até as marcações usadas nos meios digitais conhecidas como hashtag, que facilitam a pesquisa nesses ambientes virtuais.

Assim, o resgate de informações sobre cada evento e os participantes envolvidos se tornou bem mais prático e seletivo, pois pode-se selecionar as notícias sobre cada uma das edições já realizadas. Dessa maneira vemos a importância historiográfica do evento e dos elementos culturais associados ao Piauilismo, pois as imagens divulgadas nos meios digitais tornam-se numa espécie de catálogo virtual, ressaltando que a memória do patrimônio cultural trabalhado mantenha-se viva. "As memórias são sempre construídas no presente, a respeito de um passado, mas que se ancora no futuro". (HENRIQUES, 2017, p.128).

O resgate do patrimônio cultural abordado pelo Piauilismo mostra as diversas possibilidades da criação do futuro designer de moda que transforma elementos imagéticos, sensoriais e a oralidade que explora os mitos e lendas locais que povoam o ambiente da imaterialidade cultural, caracterizado muitas vezes pelo intangível, torna-se em algo tangível, através da indumentária.

"A tarefa do historiador da indumentária é, sobretudo, colher, caracterizar e transmitir a relação que, nos diferentes períodos, os homens e as mulheres estabeleceram e mantiveram com as roupas, os acessórios, os ornamentos e as cores." (MUZZARELLI, 2008, p. 28). E o registro nas redes sociais sobre o processo de construção da imagem a ser trabalhada em cada edição evidencia o resgate através da pesquisa e que elementos da matéria prima que mostra o valor do regional. 


\section{MÍDIAS DIGITAIS COMO MEIO DE PRESERVAÇÃO PATRIMONIAL}

O que são as mídias digitais? Para que elas servem? Como podemos preservar o patrimônio cultural através das mídias digitais? Qual a importância das mídias digitais para a preservação da memória ou do patrimônio cultural? São inúmeras as perguntas acerca dessa memória digital através das redes sociais e sites.

As mídias digitais são todos os meios de comunicações feitos por meio da internet, "Criar conteúdo para páginas, sites e canais também faz parte do processo de criação nas mídias digitais. Essas ações estreitam o relacionamento com usuários e criam vínculos entre empresa e cliente, algo extremamente importante no Inbhoud Marketing". (PROJETUAL COMUNICAÇÃO, 2013, s/p).

Para entender melhor, o Inbhoud Marketing é uma estratégia utilizada na internet em que consiste na produção e compartilhamento de conteúdos digitais de qualidade, com a intenção de atingir um público-alvo específico, a partir das estratégias de marketing digital. Servindo para disseminar informações acerca de produtos e eventos, neste caso utilizado para divulgar a produção do evento e o próprio Piauilismo.

Com as dez edições do Piauilismo disponibilizadas em especial pelo próprio site da UFPI, temos os registros realizados pela instituição, como forma de preservar a memória das produções por ela realizada, além disso tem-se link de acesso para o canal UFPI TV, no Youtube, possibilitando visualização do vídeo com relatos dos professores envolvidos, tais como Ascânio Wanderley, Maria de Jesus, Gizela Falcão e Artemísia Caldas que estiveram trabalhando em conjunto com os discentes na produção e curadoria do projeto de extensão do evento institucional.

Além do site da UFPI, tem-se acesso as informações do evento pelo Facebook que se tornou uma ferramenta bastante importante, principalmente para as 9 (nove) primeiras edições do Piauilismo, por ser utilizado pelos discentes responsáveis pela divulgação do evento, como o compartilhamento de pessoas que assistiram ao evento ou participaram como modelo ou patrocinador contribuindo para um conteúdo historiográfico do evento a se tornar bem coeso e extenso. Outra fonte de divulgação que está em sendo utilizada desde a sétima edição do Piauilismo é o Instagram, mecanismo que foi

[...], criado em outubro de 2010, é a rede social online mais utilizada no mundo para compartilhar imagens e vídeos e, por este traço, ideal para o mercado da moda. Em função da portabilidade e ubiquidade, a plataforma ressignificou o uso das câmeras no celular e a tendência mobile no consumo. É estratégica para trabalhar com os desejos e aspirações 
das pessoas e tem se mostrado eficiente na criação da(s) identidade(s) visual(is) dos profissionais e empresas. (HINERASKY, 2014, p.3)

Possibilitando o acesso as informações das produções artísticas da divulgação do Piauilismo, além de estimular profissionais de diversas áreas da moda, gastronomia e demais produtos que queiram trabalhar como parceiros em cada evento realizado em diversos pontos turísticos da cidade de Teresina, como no Palácio de Karnak, Clube dos Diários, Theatro 4 (quatro) de Setembro, Praça de Evento do Shopping Riverside Walker, Espaço de Eventos do edifício garagem do Teresina Shopping nas dez edições realizadas, esses foram os espaços utilizados e divulgadores de patrimônio material e veículos de divulgação através do marketing dos grandes e médios lojistas envolvidos nos patrocínios em parceria com a UFPI, através dos coordenadores do evento.

As narrativas registradas nas redes sociais funcionam como peças que se encaixam, como uma espécie de quebra-cabeça, que possibilitam a leitura de uma memória social através das junções de informações disponíveis no ambiente virtual, seja por meio das fotografias ou vídeos do Facebook, Instagram e Youtube. Tem-se outra ferramenta que possibilita a comunicação, divulgação e estratégias para o evento com parceiros comerciais e participantes do projeto através do WhatsApp. No entanto, este último recurso é restrito ao grupo que participa e não sendo acessível ao grande público.

\section{DISCUSSÃO E RESULTADOS}

O Piauilismo vem crescendo a cada edição, tornou-se um evento esperado por todos da comunidade acadêmica e teresinense, de certa forma este projeto tem impulsionado o mercado local, seja com a divulgação dos novos designers, com a divulgação das empresas parceiras e patrocinadoras, pois o evento é de grande porte e os patrocinadores ajudam em parte do orçamento, outro ponto importante é mostrar a valorização do patrimônio local através da música, cores, formas, texturas, matéria-prima utilizada na confecção dos acessórios e até mesmo da indumentária apresentada no desfile, pois há a participação de músicos locais, músicas regionais, grupos de dança que mostram um pouco da arte popular, com o Bumba-meu-boi e até arte contemporânea com performances. Um mix de erudito e popular em todo o contexto trabalhado nesta produção.

Outro ponto a ser destacado com a valorização desta memória é falar sobre o e-book "Piauilismo: por trás da criação" que a turma da nona edição, do 
Piauilismo, em conjunto com os professores Ascânio Wanderley e Francisca Danielle organizaram dados importantes sobre a construção da identidade do evento, mostram desde a pesquisa sobre os elementos da fauna, flora e mitos piauienses que foram usados no briefing e como a peça da coleção autoral de cada discente foi elencado os elementos do patrimônio local, sendo assim, um outro recurso digital que registra e resgata a memória do Piauí através do Piauilismo.

\section{CONSIDERAÇÕES FINAIS}

Com a efemeridade do mundo contemporâneo vale destacar que a utilização da pesquisa de campo, bibliográfica e digital possam dar respaldo e visibilidade aos assuntos pertinentes a memória seja ela como o patrimônio cultural piauiense com elementos que caracterizam o saber do povo, através do modo de se fazer, a oralidade e as produções de objetos, que destacamos nas produções das indumentárias produzidas pelos novos designers do curso de Moda, Design e Estilismo da UFPI que resgatam a memória que muitas vezes é perdida pelo seu povo.

Vale-se ressaltar que na contemporaneidade o acesso à internet e aos buscadores onlines, assim como o Google, para saber ou "relembrar", já que a memória humana não preserva com exatidão os fatos para serem descritos, as mídias digitais auxiliam nessa volta ao passado, fazendo-o reviver os momentos que ele fez parte do processo de construção da identidade do povo piauiense, assim como os registros acerca das edições anteriores e quem sabe futuras produções do Piauilismo, mostrando o modo de ver o mundo que os cercam através da moda.

\section{REFERÊNCIAS}

CARVALHO, Ascânio Wanderlei de. SOUSA, Francisca Danielle Araújo de. (org.). Piauilismo: Por trás da criação. 1. ed. Teresina: EDUFPI, 2019. E-Book. ISBN 978-85-509-0451-1. Disponível em: http://www.ufpi.br/arquivos_download/arquivos/EDUFPI/Ebook_Piauilismo_por_tr\%C3\%A1s_ da_cria\%C3\%A7\%C3\%A3o20190523164755.pdf. Acesso em: 22/ jul/ 2019.

CHOAY, Françoise. A alegoria do patrimônio. São Paulo: Estação Liberdade: Editora UNESP, 2001. 
HENRIQUES, Rosali Maria Nunes. Narrativas, Patrimônio digital e preservação da memória no Facebook, Revista Observatório, Palmas, v. 3, n. 5, p. 123 - 146, 1/ago/2017.

HINERASKY, Daniela Aline. O Instagram Como Plataforma De Negócio de Moda: dos "itbloggers" às "it-marcas”. In: Congresso Internacional Comunicação e Consumo - COMUNICON 2014., São Paulo. Anais, São Paulo: PPGCOM ESPM, 2014. p. 3. Disponível em: http://www3.espm.br/download/ Anais_Comunicon_2014/gts/gt_seis/GT06_HINERASKY.pdf. Acesso em: 29/ jul/ 2019.

JONES, Sue Jenkyn. Fashion design - o manual do estilista. 3.ed. São Paulo: Cosac Naif, 2011.

MATHARU, Gurmit. O que é design de moda? Porto Alegre: Bookman, 2011.

MUZZARELLI, Maria Giuseppina. Um outro par de mangas In PAOLO, Sorcinelli. Estudar a moda: corpos, vestuários, estratégias. São Paulo: Editora Senac São Paulo, 2008. p.19 - 29.

PROJETUAL COMUNICAÇÃO. Cases e News. Redes sociais, mídias sociais ou mídias digitais? Qual é a diferença?. 06 de maio de 2013. Disponível em: https://projetual.com.br/redes-sociais-midias-sociais-ou-midias-digitais-qual-e-a-diferenca/. Acesso em: 30/ jul/ 2019. 